\title{
A case series of emtricitabine-induced pure red cell aplasia
}

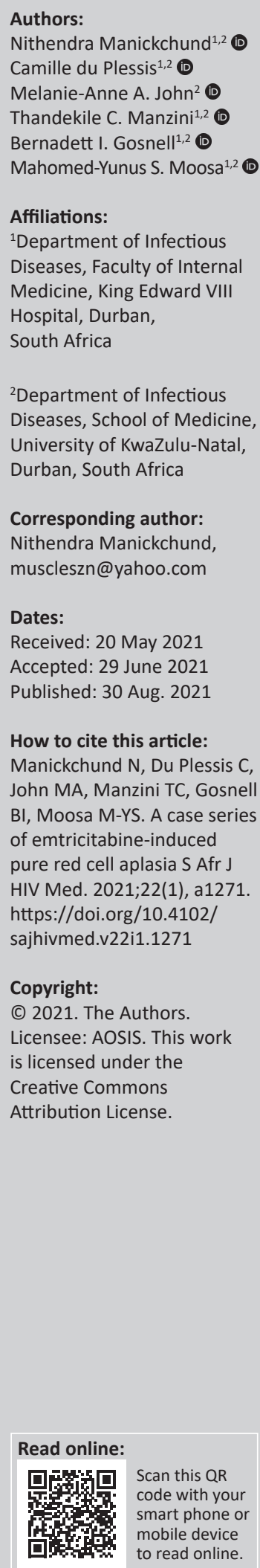

Background: Anaemia is common in patients with retroviral disease. New or worsening anaemia after initiation of antiretroviral (ARV) treatment has a broad differential diagnosis.

Objectives: We describe six patients who developed transfusion-dependent anaemia on first-line therapy (tenofovir, emtricitabine and efavirenz) and, by exclusion, implicated emtricitabine in the aetiology of the anaemia.

Method: We conducted a retrospective chart review of patients seen at the Infectious Diseases specialist clinic at King Edward VIII Hospital in KwaZulu-Natal between 2014 and 2016. We focused on patients with isolated, refractory and transfusion-dependent anaemia occurring after initiation of ARVs, in whom bone marrow biopsies were consistent with pure red cell aplasia (PRCA) without an identifiable secondary cause.

Results: All the patients were female, with a median (range) age and baseline CD4 cell count of 42.5 (23-61) years and 237 (83-329) cells $/ \mathrm{mm}^{3}$, respectively. Before presenting with symptomatic anaemia, the duration on emtricitabine was 4.5 (2-8) months. At presentation, all patients had an HIV viral load of $<1000$ copies $/ \mathrm{mL}$ and a CD4 cell count of 314 (213-389) cells $/ \mathrm{mm}^{3}$. The median time to recovery following the discontinuation of emtricitabine was 2 (1-4) months. After a median of 12 months, all patients were successfully rechallenged with emtricitabine and remained well for a follow-up period of 24 (7-36) months.

Conclusion: This study provides strong circumstantial evidence that emtricitabine plays an important role in the pathogenesis of reversible PRCA. The mechanisms through which emtricitabine induces PRCA remain unclear and require further study.

Keywords: emtricitabine; pure red cell aplasia; drug induced; rare drug toxicity; adverse drug reaction; antiretroviral; anaemia.

\section{Introduction}

Anaemia is common in persons living with HIV (PLWH). New onset or worsening anaemia on initiation of antiretroviral treatment (ART) has a wide differential diagnosis. Toxicity from antiretroviral drugs such as the nucleoside analogues zidovudine (AZT) and lamivudine (3TC) must be considered.

In 1998, several case reports implicated 3 TC as a cause of pure red cell aplasia (PRCA)., ${ }^{1,3,4,5}$ Initially, 3TC was thought to potentiate the haematological toxicity of AZT, following reports of two patients who presented with profound anaemia while on both drugs. ${ }^{1}$ Later, 3TC was shown to cause anaemia independently of AZT. ${ }^{2}$

There are several similarities between 3TC and emtricitabine (FTC). Both are cytosine deoxyribonucleoside analogues that target the HIV reverse transcriptase enzyme by acting as a chain terminator of viral DNA synthesis, with the only difference in their chemical structure being a fluorine atom bound to carbon 5 of the FTC pyrimidine ring (Figure 1). ${ }^{6,7}$ They share similar toxicities, are active against the hepatitis B virus and select the same resistance mutations. Emtricitabine, however, has a longer half-life, a higher binding affinity for the reverse transcriptase enzyme and demonstrates a greater synergy with tenofovir in inhibiting HIV replication. 6,8 The most common mechanism of nucleoside reverse transcriptase inhibitor (NRTI) toxicity is inhibition of the human mitochondrial DNA polymerase $\gamma$ enzyme, which leads to mitochondrial dysfunction. The structural differences between FTC and 3TC reduce the affinity of FTC for the $\gamma$ polymerase enzyme 100 -fold. ${ }^{9}$ This likely explains the reduced toxicity of FTC compared to 3TC. ${ }^{9}$ 


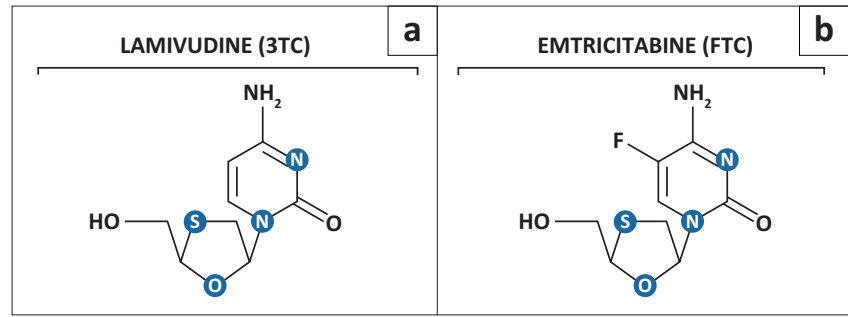

Source: Please see the full reference list of the article, Pharmaceutical equivalence $\&$ clinical interchangeability lamivudine \& emtricitabine. Supplementary section to the 2013 WHO consolidated guidelines on the use of antiretroviral drugs for treating and preventing HIV infection. Chapter 7 - Antiretroviral therapy. Geneva: WHO 2013 [cited 2019 Mar 20], p. 32-36. Available from: https://www.who.int/hiv/pub/ guidelines/arv2013/arv2013supplement_to_chapter07.pdf?ua=1, for more information FIGURE 1: The chemical structure of (a) lamivudine and (b) emtricitabine.

Because of the striking similarities between 3TC and FTC and the development of severe anaemia soon after ART initiation, in the absence of another cause, we suspected that FTC might be responsible for the PRCA. We present observational data from six patients with probable FTC-induced transfusiondependent anaemia following the initiation of a fixed-dose combination of tenofovir, FTC and efavirenz.

\section{Method}

This study was based at the Infectious Diseases specialist clinic at King Edward VIII Hospital, KwaZulu-Natal. We conducted a retrospective chart review of all patients presenting to the clinic between 2014 and 2016 with a haemoglobin $(\mathrm{Hb})$ level of $<7 \mathrm{~g} / \mathrm{dL}$ following the initiation of first-line ART. ${ }^{10}$ The department has a long-standing interest in PLWH with PRCA secondary to parvovirus B19 infection. As a result, patients with unexplained, transfusiondependent anaemia are actively recruited for further evaluation.

Patients were included if they were aged $>18$ years, infected with HIV, on an FTC-containing regimen and had isolated anaemia with a bone marrow examination consistent with PRCA, without an alternate explanation. There had to be a temporal relationship between anaemia and the use of FTC with at least 3 months' follow-up at the clinic.

All patients underwent comprehensive investigations to determine the cause of their anaemia, including iron studies, folate and vitamin B12 levels, viral studies, a connective tissue screen and an examination of the bone marrow. The information captured included the baseline and most recent CD4 cell counts, HIV viral load, previous history of ART, comorbidities and concomitant medication use, including herbal medication. Secondary causes of red cell aplasia were excluded clinically and by directed laboratory investigations. These included pregnancy, thymoma, lymphoma, systemic lupus erythematosus, rheumatoid arthritis, juvenile chronic arthritis, multiple endocrine neoplasms and drugs such as AZT and 3TC. Infection with the following viruses was excluded: parvovirus B19, Epstein-Barr virus, human T-cell lymphotropic virus 1 , and hepatitis A, B and C. Bone marrow aspirate and trephine biopsies were performed on all patients.
Patients were considered for a rechallenge with FTC once they became transfusion independent. For the rechallenge, the following criteria had to be met:

- Stable normal $\mathrm{Hb}$ for at least 3 months

- Fully suppressed HIV viral load for at least 3 months

- Informed consent from the patient.

\section{Ethical considerations}

This study was approved by the University of KwaZuluNatal Biomedical Research Ethics Committee (BREC; reference number BE586/17). This was a retrospective chart review. Because of the study design, patients' consent was not required.

\section{Results}

Six patients met the inclusion criteria. All were African and female, with a median (range) age of 43 (35-61) and CD4 cell count of 237 (83-329) cells $/ \mathrm{mm}^{3}$. The median $\mathrm{Hb}$ at the time of ART initiation was $9 \mathrm{~g} / \mathrm{dL}$ (Table 1 ). All patients were ART naïve before the commencement of FTC. Clinically, all patients had normal nutritional status, with a median (range) serum albumin value of 33.5 (29-38) g/L. The median time to the first transfusion after the initiation of FTC-based ART was 4.5 months. All patients had very low $\mathrm{Hb}$ at the initial presentation (median: $2.65 \mathrm{~g} / \mathrm{dL}$ ), with normal white cell and platelet counts.

At the time of referral to investigate the anaemia, all patients had an HIV viral load of $<1000$ copies $/ \mathrm{mL}$. Bone marrow biopsies on all patients revealed a reduction in the erythroid series, with normal to increased myeloid series. All histopathology findings were consistent with PRCA. Bone marrow culture for mycobacteria was negative for all patients.

All patients were switched from a fixed drug combination containing FTC to single agents consisting of abacavir, tenofovir and efavirenz. Following this change, all patients made a complete recovery. The median time to recovery of the $\mathrm{Hb}$ to $10 \mathrm{~g} / \mathrm{dL}$ without blood transfusions was 2 months. Four patients did not require any further transfusions after the switch. Patient 4 required a once-off transfusion of three units of packed red blood cells within 2 weeks of the switch, and Patient 5 required a total of seven units of packed red blood cells over two admissions within the first 3 months of FTC discontinuation. Patient 6 was initially switched to abacavir (ABC) and 3TC because of a stock-out of single-agent tenofovir. Despite this, she continued to require transfusions. However, when the patient was switched to tenofovir and abacavir with permanent discontinuation of 3TC and FTC, no further blood transfusions were required, and there was a complete recovery of the $\mathrm{Hb}$.

After stable recovery of the $\mathrm{Hb}$ and consistent good control of the HIV disease monitored over a range of 4 months to 16 months (median: 12 months), all patients were offered a rechallenge with FTC as a component of a fixed drug 


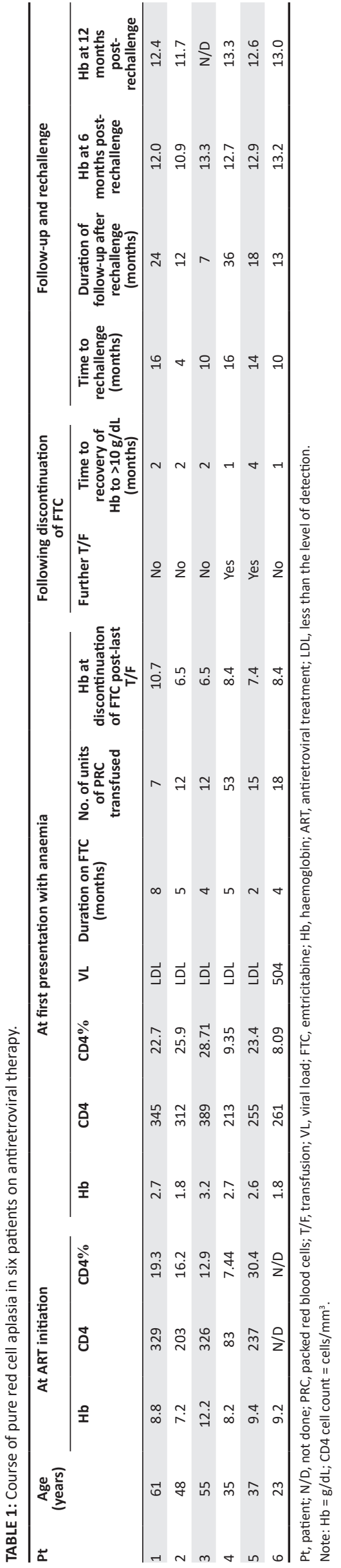

combination. All patients tolerated the rechallenge without consequence with up to 36 months of follow-up (median: 24 months; range: 7-36 months) (Table 1).

\section{Discussion}

Four fundamental factors are usually considered when implicating a drug as a causal agent of an adverse event: (1) a temporal relationship between commencing a drug and the onset of the adverse reaction, (2) biological plausibility, (3) a relationship between discontinuation of the drug and the resolution of toxicity and (4) recurrence of the toxicity on rechallenge. ${ }^{11}$ Based on at least three of these four factors, this case series provides a compelling argument for an association between FTC, a commonly used NRTI, and the development of reversible PRCA. It is not surprising that rare toxicities only manifest in clinical practice following widespread use of the drug. This underscores the importance of ongoing pharmacovigilance after introducing a drug into clinical practice (Phase IV studies). ${ }^{12}$

Our findings build on a recent report of four cases from Cape Town, South Africa, implicating FTC as the cause of transfusion-dependent anaemia. ${ }^{13}$ Of interest, the patients in that report were all female with a median time to toxicity of 3 months, similar to our findings of 4.5 months. Interestingly, the PRCA described with the related drug 3TC has been noted in male and female patients. $2^{3,3}$

Patient 5 was previously published as a case report. ${ }^{14}$ This patient's persistent anaemia was initially thought to be the result of parvovirus B19 infection based on a positive parvovirus B19 PCR test. Despite multiple rounds of intravenous immunoglobulin and attaining an undetectable HIV viral load with significant recovery of the CD4 cell count from 83 cells $/ \mathrm{mm}^{3}$ to 237 cells $/ \mathrm{mm}^{3}$, this patient remained transfusion dependent. She received 53 units of packed red blood cells over 11 months. Impressively, within 2 months of discontinuing FTC, her $\mathrm{Hb}$ improved to $11.5 \mathrm{~g} /$ $\mathrm{dL}$ and remained normal at the last follow-up, 2 years later. This case illustrates the importance of considering other entities when a suspected disease does not behave as expected. It is worth noting that this patient's parvovirus B19 PCR cycle threshold, performed several times, varied between 32.97 and 37.38 cycles, suggesting a low viral load, which in retrospect was more consistent with infection than the disease..$^{15}$ Of interest, her parvovirus B19 PCR assay remains positive more than a year after recovery at similar cycle thresholds, further supporting the unlikely role of this virus in her anaemia.

The response of Patient 6 to treatment manipulation is worth noting. The initial switch to ABC and 3TC did not substantively impact the anaemia or transfusion requirements; however, following a switch to abacavir and TDF, there was a prompt and complete recovery of the anaemia, with no further transfusions required. This suggests cross toxicity between FTC and 3TC, which is not surprising and is consistent with other reports. ${ }^{13}$ 
Several factors led us to consider rechallenging the patients with FTC after full recovery. These included the following: our observation of a prompt recovery following removal of the offending drug; FTC playing an important role in the three-drug combination treatment of HIV; FTC being a common component of most fixed drug combinations available in the state ${ }^{6,16}$; and frequent stock-outs of single agents, meaning that an uninterrupted supply of alternates to FTC would be a challenge.

Failure of the recurrence of the toxicity following rechallenge makes for interesting speculation on the pathogenesis. We posit that the marrow requires a double hit, the first being bone marrow that has been made vulnerable by preexisting anaemia or uncontrolled HIV with immune dysregulation, and the second hit being the drug, which, together with the appropriate host genetic background, leads to toxicity. Uneventful rechallenge following adequate recovery of the bone marrow suggests that the presumed genetic predisposition is necessary but not sufficient for the toxicity to manifest and that uncontrolled viral replication or immune dysregulation play an important role.

The limitations of this report include its retrospective design and small case numbers. The inferences about causality are strengthened by the temporal relationships with onset and recovery of the toxicity; however, it is important to note that this remains an uncontrolled observation.

\section{Conclusion}

This study provides strong circumstantial evidence that FTC plays an important role in the pathogenesis of reversible PRCA in a subset of patients with HIV commencing ART. This entity should be considered when common causes of refractory anaemia are excluded. The mechanisms through which FTC induces PRCA remain unclear and require further study.

\section{Acknowledgements Competing interests}

The authors declare that they have no financial or personal relationships that may have inappropriately influenced them in writing this article.

\section{Authors' contributions}

N.M. contributed to the literature search, data collection, analysis, interpretation and writing. M.-Y.S.M. contributed to the analysis, interpretation, writing and editing. B.I.G. and C.D.P. contributed to the data collection, analysis and editing. M.-A.A.J. and T.C.M. contributed to the analysis and interpretation and editing.

\section{Funding information}

This research received no specific grant from any funding agency in the public, commercial or not-for-profit sectors.

\section{Data availability}

Data sharing is not applicable to this article as no new data were created or analysed in this study.

\section{Disclaimer}

The views and opinions expressed in this article are those of the authors and do not necessarily reflect the official policy or position of any affiliated agency of the authors.

\section{References}

1. Hester EK, Peacock JE. Jr. Profound and unanticipated anemia with lamivudinezidovudine combination therapy in zidovudine-experienced patients with HIV infection. AIDS. 1998;12(4):439-440.

2. Weitzel T, Plettenberg A, Albrecht $D$, Lorenzen $T$, Stoehr A. Severe anemia as a newly recognized side-effect caused by lamivudine. AIDS. 1999;13(16):2309-2311. https://doi.org/10.1097/00002030-199911120-00018

3. Majluf-Cruz A, Luna-Castanos G, Trevino-Perez S, Santoscoy M, Nieto-Cisneros L. Lamivudine-induced pure red cell aplasia. Am J Hematol. 2000;65(3):189-191. https://doi.org/10.1002/1096-8652(200011)65:3\%3C189::AID-AJH2\% 3E3.0.CO;2-6

4. Nakamura K, Tateyama M, Tasato D, et al. Pure red cell aplasia induced by lamivudine without the influence of zidovudine in a patient infected with human immunodeficiency virus. Intern Med. 2014;53(15):1705-1708. https://doi. org/10.2169/internalmedicine.53.2460

5. John MA, Rhemtula YA, Menezes CN, Grobusch MP. Lamivudine-induced red cell aplasia. J Med Microbiol. 2008;57(Pt 8):1032-1035. https://doi.org/10.1099/ jmm.0.47782-0

6. Pharmaceutical equivalence \& clinical interchangeability lamivudine \& emtricitabine. Supplementary section to the 2013 WHO consolidated guidelines on the use of antiretroviral drugs for treating and preventing HIV infection Chapter 7 - Antiretroviral therapy. Geneva: WHO; 2013 [cited 2019 Mar 20] p. 32-36. Available from: https://www.who.int/hiv/pub/guidelines/arv2013/ arv2013supplement_to_chapter07.pdf?ua=1

7. Scaglione F, Berrino L. Cytosine deoxyribonucleoside anti-HIV analogues: A smal chemical substitution allows relevant activities. Int I Antimicrob Agents. 2012;39(6):458-463. https://doi.org/10.1016/j.ijantimicag.2011.11.013

8. Ford N, Shubber Z, Hill A, et al. Comparative efficacy of lamivudine and emtricitabine: A systematic review and meta-analysis of randomized trials. PLoS One. 2013;8(11):e79981. https://doi.org/10.1371/journal.pone.0079981

9. Sohl CD, Szymanski MR, Mislak AC, et al. Probing the structural and molecular basis of nucleotide selectivity by human mitochondrial DNA polymerase $\gamma$. Proc Natl Acad Sci USA. 2015;112(28):8596-8601. https://doi.org/10.1073/pnas. 1421733112

10. WHO. Haemoglobin concentrations for the diagnosis of anaemia and assessment of severity. Vitamin and Mineral Nutrition Information System. Geneva: World Health Organization; 2011 [cited 2020 Aug 26]. Available from: http://www.who.int/vmnis/indicators/haemoglobin

11. Pande S. Causality or relatedness assessment in adverse drug reaction and its relevance in dermatology. Indian J Dermatol. 2018;63(1):18-21. https://doi. org/10.4103/ijd.IJD_579_17

12. Bourin $\mathrm{M}$, Chagraoui A. What value for the phase IV clinical trials? SOJ Pharm Pharm Sci. 2016;3(2):1-3. https://doi.org/10.15226/2374-6866/3/2/00138

13. Cohen K, Viljoen $C$, Njuguna $C$, Maartens G. Emtricitabine-associated red cell aplasia. AIDS. 2019;33(6):1095-1096. https://doi.org/10.1097/OAD.000000000000 2136

14. Manickchund N, Du Plessis C, John MA, et al. Emtricitabine-induced pure red cell aplasia. S Afr J HIV Med. 2019;20(1):983. https://doi.org/10.4102/sajhivmed. v20i1.983

15. Young NS, Brown KE. Parvovirus B19. N EngI J Med. 2004;350(6):586-597. https:// doi.org/10.1056/NEJMra030840

16. Wainburg MA. The impact of the M184V substitution on drug resistance and viral fitness. Expert Rev Anti Infect Ther. 2004;2(1):147-151. https://doi.org/10. 1586/14787210.2.1.147 